\title{
Catalysis and Pore Initiation in the Anodic Dissolution of Silicon in HF
}

\author{
E. S. Kooij and D. Vanmaekelbergh \\ Department of Condensed Matter, Debye Institute, Utrecht University, 3508 TA Utrecht, The Netherlands
}

\section{ABSTRACT}

A mechanism for the (photo)anodic dissolution of silicon in HF containing solutions is proposed, which explains the dependence of both the photocurrent quantum yield and the efficiency for hydrogen evolution on the flux of absorbed photons. The model assumes that the chemical oxidation of an $\mathrm{Si}$ (II) intermediate to an $\mathrm{Si}$ (IV) product, which is accompanied by the formation of a hydrogen molecule, is catalyzed by a mobile Si(I) dissolution intermediate. The surface chemistry, corresponding to the proposed mechanism of anodic dissolution, is discussed. Furthermore, it is shown that the mechanism may provide an explanation, based on chemical kinetics, for initiation of pores during anodic etching.

\section{Introduction}

The anodic dissolution of silicon in HF-containing solutions is characterized by several remarkable features. ${ }^{1-28}$ First of all, the anodic etching reaction is a multistep process which involves charge-transfer reactions both via the valence band (hole capture) and via the conduction band (electron injection). ${ }^{2-5,7,9,14,17,18,20,22}$ In addition, hydrogen evolution has been observed at potentials far more positive than the Nernst potential for the $\mathrm{H}^{+} / \mathrm{H}_{2}$ couple. ${ }^{8,14,17,18}$ Finally, the morphology of the etched silicon surface strongly depends on the anodization conditions. ${ }^{11,13,15,16,18-20,23-26}$ For example, thick nanoporous silicon layers can be formed, which exhibit interesting optical properties, viz. strong visible photoluminescence ${ }^{11}$ and electroluminescence. ${ }^{29,30}$ Although the mechanism for pore propagation during porous silicon etching seems reasonably well understood, ${ }^{15,18,19}$ the origin of pore initiation remains a subject of speculation; ${ }^{24,26-28}$ even on ideally flat surfaces it is possible to etch very inhomogeneous, highly porous structures.

Anodic dissolution of n-type silicon occurs only at a considerable rate when the electrode is illuminated, ${ }^{1-3,8,9,22}$ which means that a valence band hole is required for the etching reaction. During dissolution of n-type silicon in HF solutions, photocurrent multiplication has been observed; more than one charge carrier per absorbed photon contributes to the photocurrent measured in the external circuit. . $^{2-5,7-9,14,17,22}$ Photocurrent quantum efficiencies $Q$ (the number of charge carriers measured per absorbed photon) of up to four have been found with n-type silicon electrodes. ${ }^{2,4,9,14,17}$ An explanation for the current quadrupling effect has been sought in terms of a model involving four consecutive electrochemical dissolution steps. ${ }^{4,9,14,17}$ In the initial step, a photogenerated hole is captured in a surface bond and a surface intermediate is formed. This intermediate is further oxidized to an Si(IV) species by three consecutive electrochemical steps, in which electrons are thermally excited to the conduction band. As a consequence, four charge carriers can be measured as photocurrent in the external circuit per absorbed photon. The photocurrent quantum efficiency $Q$ is dependent on the incident photon flux; $;^{2,4,9,14}$ at low light intensity the quantum efficiency is almost 4 , but drops to 2 as the photon flux is increased. This phenomenon has been explained by assuming that electron injection from, and hole capture by surface intermediates are competing processes in two of the three charge-transfer steps. ${ }^{4,9}$ The hole capture rate is proportional to the product of the surface hole concentration $p_{\mathrm{s}}$ and the rate constant $k_{\mathrm{p}}$, while electron injection is a thermally activated process, depending only on the rate constant $k_{n}$. At low intensity the product $k_{\mathrm{p}} p_{\mathrm{s}}$ is smaller than $k_{\mathrm{n}}$, but as the number of incident photons increases, $k_{\mathrm{p}} p_{\mathrm{s}}$ increases and, eventually, hole capture will become the predominant process.
The above mechanism has proven successful in explaining the results obtained with other semiconductors. ${ }^{31-34}$ However, during photoanodic etching of n-type silicon at sufficiently high light intensity a photocurrent quantum efficiency of 2 is found and hydrogen is evolved at a considerable rate; for each silicon atom dissolved a $\mathrm{H}_{2}$ molecule is formed. ${ }^{8,14,17,18}$ This means that the efficiency for hydrogen evolution $\eta_{\mathrm{H}_{2}}$ (the number of $\mathrm{H}_{2}$ molecules formed per absorbed photon) is 1 . Several models for the dissolution reaction have been proposed to account for these results. ${ }^{13,18,21,22,25}$ From Fourier transform infrared (FTIR) measurements it is well established that the silicon surface is mainly hydrogen-terminated $(\mathrm{Si}-\mathrm{H})$ during anodic dissolution and pore formation. ${ }^{6.12}$ Lehmann and Gösele $^{13}$ and Gerischer et al. ${ }^{22}$ proposed models for the silicon dissolution in $\mathrm{HF}$, which account for the hydrogen termination and for a photocurrent quantum yield of 2 and $\mathrm{H}_{2}$ yield of 1 as observed at high intensity. In both models it is assumed that a valence band hole is captured in an Si-H surface species, resulting in an electron-deficient $\mathrm{Si}-\mathrm{H}$ bond ${ }^{13,22}$ The main difference between the two descriptions of the surface chemistry is the position of the positive charge in the electron-deficient $\mathrm{Si}-\mathrm{H}$ bond. Lehmann and Gösele assume that the positive charge is located on the silicon side of the bond, resulting in a hydrogen radical ${ }^{13}$ Gerischer et al. assume that hole trapping in the $\mathrm{Si}-\mathrm{H}$ bond results in a silicon surface radical species and a proton. ${ }^{22}$ In both cases the hole capture step is followed by injection of an electron into the conduction band, thereby accounting for the observed photocurrent doubling. Further chemical oxidation of the divalent silicon intermediate to a tetravelent dissolution product is accompanied by the formation of one $\mathrm{H}_{2}$ molecule, in accordance with the experimental results at high light intensity. The models of Lehmnann and Gösele ${ }^{13}$ and Gerischer et $a .^{22}$ can account for the fact that the photocurrent quantum efficiency is 2 and the hydrogen yield is 1 during photoanodic dissolution of $n$-type silicon at sufficiently high light intensity.

However, Stumper and Peter ${ }^{14}$ have found, with rotating ring-disk voltammetry, that not only the photocurrent quantum efficiency, but also the efficiency for hydrogen evolution $\eta_{\mathrm{H}_{2}}$ depends on the photon flux. The experimental results are summarized in Fig. $1 .^{14}$ Hydrogen is not evolved when $Q$ is approximately 4 at low light intensity. At higher light intensity, as $Q$ decreases, hydrogen evolution is observed and the efficiency for hydrogen evolution $\eta_{\mathrm{H}_{2}}$ increases with increasing photon flux. When the photon flux is sufficiency large, $Q$ is approximately 2 and the efficiency for hydrogen evolution $\eta_{\mathrm{H}_{2}}$ is 1 . It was remarked by the authors ${ }^{14,17}$ that it is impossible to explain the light intensity-dependence of both the photocurrent quantum efficiency $Q$ and the efficiency for hydrogen evolution $\eta_{\mathrm{H}_{2}}$ (as shown in Fig. 1) with the hitherto proposed models. Both the rate of electron injection from and the rate of 


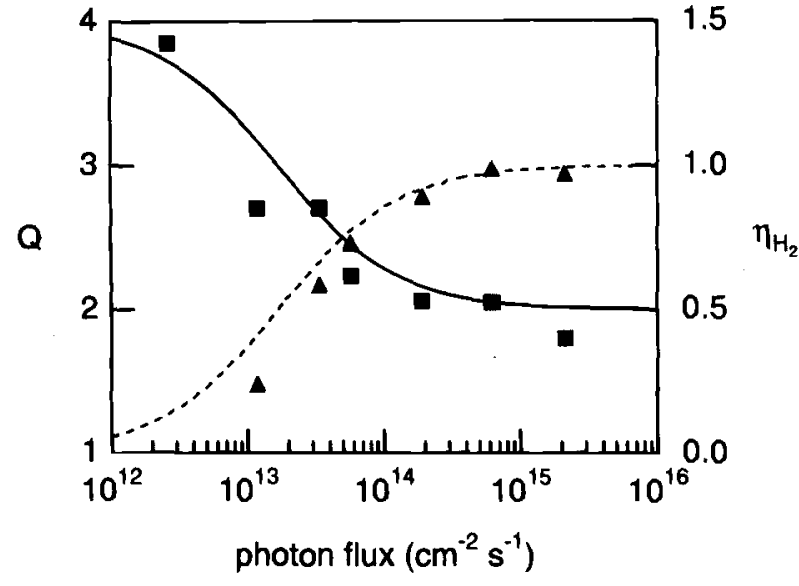

Fig. 1. Intensity-dependence of the photocurrent quantum efficiency $Q(D)$ and the efficiency for hydrogen evolution $\eta_{H_{2}}(\Delta)$ for n-type silicon in $1 \mathrm{MNH}_{4}$ F. Results were taken from Ref. 14. The calculated intensity-dependence of $Q$ (solid line) and $\eta_{\mathrm{H}_{2}}$ (dashed line) were obtained from Eq. 15 and 16 with $k_{a} /\left(k_{1} k_{2}\right)=6^{2} \times 10^{-14} \mathrm{~cm}^{2} \mathrm{~s}$.

proton reduction by a surface intermediate are simply proportional to the concentration of this intermediate. Hence, the competition between both processes is expected to be independent of the photon flux, in contrast to the experimental results (Fig. 1). The results suggest that the hydrogen evolution reaction is of higher order in the concentration of intermediate species than the electron injection process. ${ }^{14,17}$

We have reconsidered the results of Stumper and Peter ${ }^{14}$ and developed a kinetic scheme, which accounts for the observed decrease of $Q$ and the simultaneous increase of $\eta_{\mathrm{H}_{2}}$ with increasing light intensity. The key step in the kinetic model is the chemical oxidation of an Si(II) surface intermediate to an Si(IV) dissolution product with $\mathrm{H}_{2}$ formation, a reaction catalyzed by another dissolution intermediate. The surface chemistry corresponding to the kinetic model is discussed in relation to previously proposed models. Furthermore, the implications of the autocatalytic step for the dissolution process and the resulting morphology of the etched silicon surface are mentioned.

\section{Kinetic Model}

In the kinetic model, unoxidized silicon surface atoms are represented by $\mathrm{Si}(\mathrm{O})$. As mentioned in the introduction the anodic dissolution of n-type silicon in $\mathrm{HF}$ solutions only occurs under illumination, so the first step obviously involves the capture of a valence band hole by an $\mathrm{Si}(\mathrm{O})$ surface species

$$
\mathrm{Si}(\mathrm{O})+h_{\mathrm{vB}}^{+} \stackrel{k_{0}}{\rightarrow} \mathrm{Si}(\mathrm{I})
$$

We assume that the hole is captured in a Si-Si bond and the Si(I) intermediate then corresponds to an electrondeficient Si-Si back bond; hole capture in a back bond has been reported in studies of the anodic dissolution of III-V semiconductors. ${ }^{31,34}$ The assumption of an electron-deficient $\mathrm{Si}-\mathrm{Si}$ bond is different from that proposed by Lehmann and Gösele ${ }^{13}$ and Gerischer et al., ${ }^{22}$ who assumed hole capture by a Si-H bond. The photocurrent quantum efficiency is approximately 4 at very low light intensities; four charge carriers are measured in the external circuit for each photon absorbed. This can only be achieved if the initial hole capture is followed by three consecutive electron injection steps

$$
\begin{gathered}
\mathrm{Si}(\mathrm{I}) \stackrel{k_{1}}{\rightarrow} \mathrm{Si}(\mathrm{II})+e_{\mathrm{CB}}^{-} \\
\mathrm{Si}(\mathrm{II}) \stackrel{k_{3}}{\rightarrow} \mathrm{Si}(\mathrm{III})+e_{\mathrm{CB}}^{-} \\
\mathrm{Si}(\mathrm{III}) \stackrel{k_{3}}{\rightarrow} \mathrm{Si}(\mathrm{IV})+\mathrm{Si}(\mathrm{O})+e_{\mathrm{CB}}^{-}
\end{gathered}
$$

In the last step, a completely oxidized Si(IV) species is formed, which enters solution as a dissolution product
$\left(\mathrm{SiF}_{6}^{2-}\right)$ and a new, unoxidized $\mathrm{Si}(\mathrm{O})$ species is exposed at the electrode surface.

The concentration of $\mathrm{Si}(\mathrm{I})$ intermediates will increase with increasing light intensity (reaction 1 ). If this intermediate is able to move along the silicon surface, it may interact with other dissolution intermediates. The surface mobility of $\mathrm{Si}(\mathrm{I})$ is determined by the ease with which a bonding electron can pass from an unbroken Si-Si surface back bond to a neighboring electron-deficient surface bond. From work on III-V semiconductors it is known that in many cases such mobile intermediates are involved in anodic dissolution. ${ }^{31}$ To account for the decrease of the photocurrent quantum efficiency and the formation of hydrogen as the photon flux becomes larger, a reaction is required which is second order in concentration of the intermediate. We assume that an $\mathrm{Si}(\mathrm{II})$ intermediate is chemically oxidized to an Si(IV) dissolution product while two protons are reduced to $\mathrm{H}_{2}$ in a reaction which is catalyzed by an $\mathrm{Si}(\mathrm{I})$ surface intermediate. The overall reaction can be written as

$$
\mathrm{Si}(\mathrm{II})+2 \mathrm{H}^{+} \underset{\mathrm{Si}(\mathrm{I})}{\stackrel{k_{\mathrm{a}}}{\longrightarrow}} \mathrm{Si}(\mathrm{IV})+\mathrm{H}_{2}
$$

If reaction 5 competes with the electron injection step 3 the photocurrent quantum efficiency will decrease from 4 to 2 and the efficiency for hydrogen formation will increase from 0 to 1 with increasing photon flux.

The problem can be treated quantitatively. From the reactions 1-5 described above we can deduce equations which describe the time-dependence of the concentration of reaction intermediates. In the steady-state, the concentration of the intermediates is constant and we obtain the following relations

$$
\begin{gathered}
\frac{d p}{d t}=j_{\mathrm{h}} / e-k_{0} \mathrm{~s}_{0} p=0 \\
\frac{d s_{1}}{d t}=k_{0} \mathrm{~s}_{0} p-k_{1} s_{1}=0 \\
\frac{d s_{2}}{d t}=k_{1} s_{1}-k_{2} s_{2}-k_{\mathrm{a}} s_{1} s_{2}=0 \\
\frac{d s_{3}}{d t}=k_{2} s_{2}-k_{3} s_{3}=0
\end{gathered}
$$

where the concentrations of the $\mathrm{Si}(\mathrm{O}), \mathrm{Si}(\mathrm{I}), \mathrm{Si}(\mathrm{II})$, and $\mathrm{Si}(\mathrm{III})$ in termediates are denoted by $s_{0}, s_{1}, s_{2}$, and $s_{3}$, respectively. The photon flux is given by the hole current divided by the elementary charge $e$, which we denote as $j_{\mathrm{h}} / e=$ $j_{\mathrm{h}}^{\prime}$. In the analysis the contributions from hole capture processes, which may compete with the electron injection steps $2-4$, are not taken into account. The photocurrent quantum efficiency $Q$ is given by

$$
Q=\frac{j_{\mathrm{h}}^{\prime}+k_{1} s_{1}+k_{2} s_{2}+k_{3} s_{3}}{j_{\mathrm{h}}^{\prime}}
$$

and the efficiency for hydrogen evolution $\eta_{\mathrm{H}_{2}}$, which we defined as the number of $\mathrm{H}_{2}$ molecules formed per absorbed photon, is

$$
\eta_{\mathrm{H}_{2}}=\frac{k_{\mathrm{a}} s_{1} s_{2}}{j_{\mathrm{h}}^{\prime}}
$$

We now have to calculate the steady-state concentration of $\mathrm{Si}(\mathrm{I}), \mathrm{Si}(\mathrm{II})$, and $\mathrm{Si}(\mathrm{III})$ intermediates and insert these into Eq. 10 and 11 to obtain expressions for $Q$ and $\eta_{\mathrm{H}_{2}}$ as a function of $j_{\mathrm{h}}^{\prime}$

From Eq. 6 and 7 it is found that

$$
s_{1}=\frac{j_{\mathrm{h}}^{\prime}}{k_{1}}
$$


and when we insert this into Eq. 8 it follows that

$$
s_{2}=\frac{j_{\mathrm{h}}^{\prime}}{k_{2}\left(1+\frac{k_{\mathrm{a}}}{k_{1} k_{2}} j_{\mathrm{h}}^{\prime}\right)}
$$

Finally, the combination of this expression for $s_{2}$ and Eq. 9 yields an expression for the concentration of Si(III) intermediates

$$
s_{3}=\frac{k_{2}}{k_{3}} s_{2}=\frac{j_{\mathrm{h}}^{\prime}}{k_{3}\left(1+\frac{k_{\mathrm{a}}}{k_{1} k_{2}} j_{\mathrm{h}}^{\prime}\right)}
$$

The photocurrent quantum efficiency $Q$ and the efficiency for hydrogen evolution $\eta_{\mathrm{H}_{2}}$ follow directly from Eq. 10-14

$$
\begin{gathered}
Q=2+\frac{2}{1+\frac{k_{\mathrm{a}}}{k_{1} k_{2}} j_{\mathrm{h}}^{\prime}} \\
\eta_{\mathrm{H}_{2}}=\frac{\frac{k_{\mathrm{a}}}{k_{1} k_{2}} j_{\mathrm{h}}^{\prime}}{1+\frac{k_{\mathrm{a}}}{k_{1} k_{2}} j_{\mathrm{h}}^{\prime}}
\end{gathered}
$$

The two limiting cases, mentioned above, can be distinguished. At low light intensities, $\dot{j}_{\mathrm{h}}^{t}$ becomes small and we find that $Q=4$ and $\eta_{\mathrm{H}_{3}} \approx 0$. On the other hand, when the photon flux is sufficiently large $\left(j_{\mathrm{h}} / e>k_{1} k_{2} / k_{\mathrm{a}}\right)$ the photocurrent quantum efficiency decreases to 2 while the efficiency for hydrogen evolution reaches a limiting value of 1. With Eq. 15 and 16 it is now possible to evaluate $Q$ and $\eta_{\mathrm{H}_{2}}$ in the whole range of light intensity. Note that both expressions contain only one variable parameter $k_{\mathrm{a}} /\left(k_{1} k_{2}\right)$. By fitting the data of Fig. 1 to these equations with $k_{a} / k_{1} k_{2}$ as a parameter, a value $k_{\mathrm{a}} /\left(k_{1} k_{2}\right)=6 \times 10^{-14} \mathrm{~cm}^{2} \mathrm{~s}$ was found (see Fig. 1). It is clear that the calculated curves agree quite well with the experimental results. The kinetic scheme presented above explains the observed relationship between $Q$ and $\eta_{\mathrm{H}_{2}}$ in the entire range of light intensity. Taking into account that the reaction intermediates must be mobile along the surface, we have also derived expressions for $Q$ and $\eta_{\mathrm{H}_{2}}$ as a function of the photon flux, based on other second-order reactions, but none of these were able to account for the experimental results.

\section{Discussion}

In the first part of the discussion, the chemical implications of the mechanism are investigated. The importance of the mechanism for initial pore formation is then considered.

Surface chemistry.-Here, we consider the surface chemistry of a (100) silicon surface in the context of our kinetic scheme. The likely initial steps of the etching mechanism are given, after which a distinction is drawn between low and high light intensities, corresponding to the two limiting cases mentioned in the kinetic analysis. From in situ FTIR measurements on silicon electrodes in HF containing electrolytes, it was concluded that the electrode surface is to a large extent covered with hydrogen and that anodic dissolution in the region of porous silicon formation also results in a hydrogen terminated surface, ${ }^{6,12}$ The surface atoms of a (100) silicon electrode have two back bonds to deeper lying silicon atoms and two $\mathrm{Si}-\mathrm{H}$ bonds. These silicon surface atoms correspond to the $\mathrm{Si}(\mathrm{O})$ entity, introduced in the kinetic model.

Figure 2 describes the formation of an $\mathrm{Si}(\mathrm{II})$ intermediate from an $\mathrm{Si}(\mathrm{O})$ surface atom. In the first step, a valence band hole is captured by an $\mathrm{Si}(\mathrm{O})$ entity, resulting in an electron-deficient $\mathrm{Si}-\mathrm{Si}$ surface bond. As mentioned before this is distinctly different from other models, in which it is assumed that the hole capture results in an electron-deficient Si-H bond. ${ }^{13,22}$ This process of hole capture corresponds to reaction 1 of the kinetic scheme and the electron-deficient bond corresponds to the Si(I) intermediate. It is assumed that this $\mathrm{Si}(\mathrm{I})$ intermediate is mobile within the layer of surface back bonds. Electron transfer between neighboring Si-Si bonds may not be the only process occurring. It is also possible that an electron is transferred from one of the $\mathrm{Si}-\mathrm{H}$ surface bonds to the Si-Si bond, which yields an electron-deficient $\mathrm{Si}-\mathrm{H}$ species, similar to that mentioned by Lehmann and Gösele $^{13}$ and Gerischer et al. ${ }^{22}$ There will probably be an equilibrium between the two aforementioned cases, as denoted in Fig. 2 by the double arrow. In the following step, corresponding to reaction 2 of the kinetic scheme, an electron is thermally excited to the conduction band, while<smiles>[SiH3][SiH2][SiH2][SiH]([SiH3])[SiH2][SiH3]</smiles><smiles>[SiH3][SiH2][SiH2][SnH][SiH2][SiH3]</smiles>

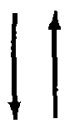

(reaction 1)<smiles>[SiH3][SiH2][SiH2][SiH3][SiH3]</smiles>

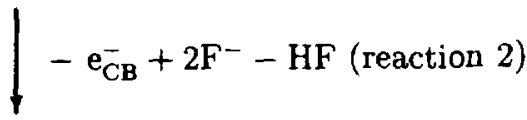<smiles>F[Si]([SiH3])([SiH2][SiH3])[SiH2][SiH2][SiH3]</smiles><smiles>[SiH3][SiH2][SiH2][SiH3]</smiles>

$[\mathrm{Si}(\mathrm{II})]$

Fig. 2. Schemotic representation of the first steps of the mecha nism for onodic dissolution of $n$-type (100) silicon in HF solutions. 
a fluorine ion bonds to the surface silicon atom. The attack of one of the $\mathrm{Si}-\mathrm{Si}$ back bonds by $\mathrm{HF}$ is the final step in Fig. 2. The Si(II) intermediate is hence a silicon atom bonded to another silicon atom, two fluorine atoms and one hydrogen atom.

Figure 3 shows the further steps of the dissolution mechanism, which are assumed to occur at low light intensity. In this case the electron injection step 3 dominates over the catalyzed reaction 5 . The $\mathrm{Si}-\mathrm{H}$ bond on the $\mathrm{HSiF}_{2}$ species, which is still attached to the surface, is destabilized due to the strongly polarized $\mathrm{Si}-\mathrm{F}$ bonds as well as the interaction with the solution. Oxidation of this destabilized $\mathrm{Si}-\mathrm{H}$ species is accompanied by electron injection into the conduction band. The two electron excitation steps of Fig. 3 correspond to reactions 3 and 4 of the kinetic scheme. Deprotonation and bonding with a fluorine ion transforms $\mathrm{HSiF}_{2}$ into $\mathrm{SiF}_{3}$. The high electronegativity of fluorine induces a strong polarization in the Si-Si back bond. Attack on this back bond by an HF molecule (final step of Fig. 3) yields an $\mathrm{SiF}_{4}$ molecule, which is further complexed by two fluorine ions forming the soluble $\mathrm{SiF}_{6}^{2-}$ species and a new $\mathrm{Si}-\mathrm{H}$ surface bond. The net result of the reactions shown in Fig. 2 and 3 for low photon flux can be represented by reaction 17

$$
\mathrm{Si}(\mathrm{O})+h_{\mathrm{VB}}^{+}+6 \mathrm{~F}^{-} \longrightarrow \mathrm{SiF}_{6}^{2-}+3 e_{\mathrm{CB}}^{-}
$$

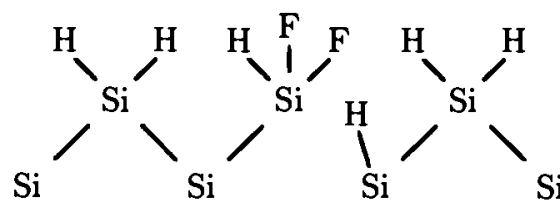

$$
\downarrow-e_{\text {CB }}^{-}-H^{+} \quad(\text { reaction } 3)
$$<smiles></smiles>

$$
\mid-\mathbf{e}_{\mathrm{CB}}^{-}+\mathrm{F}^{-}
$$

(reaction 4)<smiles>F[Si](F)([SiH3])[SiH2][SiH2][SiH3]</smiles><smiles>[3H][Hg][Si](F)(F)F</smiles>

$[\mathrm{Si}(\mathrm{IV})]$

Fig. 3. Schematic representation of the further steps of the mechanism for anodic dissolution of n-type (100) silicon in HF solutions at low light intensity.
From this it is clear that the quantum efficiency is 4, while no hydrogen gas is evolved, in agreement with the experimental results and the kinetic model

Peter et $a l .{ }^{9}$ performed a detailed study of the kinetics of the electron injection steps during photoanodic dissolution of n-type silicon in $\mathrm{NH}_{4} \mathrm{~F}$ solutions of $\mathrm{pH}$ 4. They found that the rate constants of the subsequent electron injection steps decrease considerably with increasing degree of oxidation of the intermediate: $k_{1}=2 \times 10^{4} \mathrm{~s}^{-1}$, $k_{2}=500 \mathrm{~s}^{-1}, k_{3}=0.5 \mathrm{~s}^{-1}$. This seems to be in accordance with the surface chemistry proposed in Fig. 2 and 3. The first electron injection step involves a surface radical with only one Si-F bond, the second and third injection steps involve intermediates with $2 \mathrm{Si}-\mathrm{F}$ bonds. It is reasonable to assume that the high electronegativity of fluorine decreases the rate of thermally activated electron injection. Furthermore, it must be remarked that the Si(II) intermediate proposed in Fig. 2 and 3 is relatively stable with respect to anodic charge-transfer processes.

At high light intensity, the concentration of photogenerated holes becomes large and the surface concentration of $\mathrm{Si}(\mathrm{I})$ intermediates increases (Eq. 12). In Fig. 4 likely steps of the etching mechanism at high photon flux are given. Due to the higher $\mathrm{Si}(\mathrm{I})$ concentration, the probability that this mobile intermediate interacts with an $\mathrm{Si}_{-} \mathrm{SiHF}_{2}$ [Si(II)] species becomes significant. If this is the case, an electron from the Si-Si bond, linking the $\mathrm{HSiF}_{2}$ to the surface, may be transferred to the electron-deficient interme-<smiles>[SiH3][SiH2][SiH2][SiH2][SiH3]</smiles>

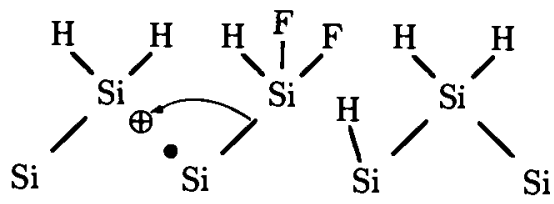<smiles>[AsH3]</smiles>
$\mathrm{Si}(\mathrm{II})$

(reaction 5)

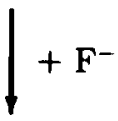<smiles>[SiH3][SiH2][SiH2][SiH3]</smiles><smiles>C[PH3+]</smiles><smiles>[SiH3][SnH2][SiH3]</smiles><smiles>[SiH3][SiH3][SiH3]</smiles>

$[\mathrm{Si}(\mathrm{I})]$

Fig. 4. Schematic representation of the further steps of the mechanism for anodic dissolution of n-type (100) silicon in HF solutions at high light intensity. 
diate. If a fluorine ion is bound to the outermost silicon atom in this process (the second step in Fig. 4), an $\mathrm{HSiF}_{3}$ molecule is detached from the surface and an unpaired electron is left behind on one of the new surface atoms. The formation of this $\mathrm{HSiF}_{3}$ intermediate has also been proposed by Lewerenz et $a l^{4}{ }^{4}$ and Peter et al. ${ }^{6}$ It was, however, impossible to detect this species with in situ FTIR experiments. ${ }^{12}$ In the last step, the surface radical reacts with a proton and the $\mathrm{Si}(\mathrm{I})$ intermediate is restored. The silicon surface remains fully hydrogenated, in agreement with experimental evidence. ${ }^{12,17}$ In the reactions of Fig. 4, no free charge carriers are involved, in contrast to what is proposed for low light intensity. If we summarize the reactions in Fig. 2 and 4 the following overall equation for the anodic dissolution of silicon in HF solutions is obtained

$$
\mathrm{Si}(\mathrm{O})+h_{\mathrm{VB}}^{+}+3 \mathrm{~F}^{-}+\mathrm{H}^{+} \stackrel{\mathrm{Si}(\mathrm{I})}{\longrightarrow} \mathrm{HSiF}_{3}+e_{\mathrm{CB}}^{-}
$$

In this reaction the $\mathrm{Si}(\mathrm{I})$ intermediate participates but is not consumed, so it can be regarded as a surface catalyst for the anodic etching reaction at higher light intensity. Only one electron is injected for each hole consumed, which implies a quantum efficiency of 2 . Due to the strong polarization of the $\mathrm{Si}-\mathrm{H}$ bond in the $\mathrm{HSiF}_{3}$ molecule, which is formed in the final reaction of Fig. 4 , it is easily attacked by HF to form hydrogen

$$
\mathrm{HSiF}_{3}+\mathrm{HF}+2 \mathrm{~F}^{-} \longrightarrow \mathrm{SiF}_{6}^{2-}+\mathrm{H}_{2}
$$

The $\mathrm{SiF}_{4}$ is further complexed by 2 fluorine ions, again forming the soluble $\mathrm{SiF}_{6}^{2-}$ species. The sum of Eq. 18 and 19 gives the overall anodic dissolution reaction occurring at sufficiently high light intensity

$$
\mathrm{Si}(\mathrm{O})+h_{\mathrm{VB}}^{+}+6 \mathrm{~F}^{-}+2 \mathrm{H}^{+} \stackrel{\mathrm{Si}(\mathrm{I})}{\longrightarrow} \mathrm{SiF}_{6}^{2-}+e_{\mathrm{CB}}^{-}+\mathrm{H}_{2}
$$

From Eq. 20 it is evident that the efficiency for hydrogen evolution $\eta_{\mathrm{H}_{2}}$, defined as the number of $\mathrm{H}_{2}$ molecules evolved per absorbed photon, amounts to 1 and that the photocurrent quantum yield is 2 . This is in agreement with the kinetic model and the experimental results at high light intensity. Reaction 20 involves a sequence of (electro)chemical steps. It follows from our kinetic analysis that the step in which a relatively stable Si(II) intermediate reacts with an $\mathrm{Si}(\mathrm{I})$ intermediate must determine the rate of the self-catalyzed branch of the dissolution mechanism, which competes with electron injection from $\mathrm{Si}(\mathrm{II})$, in order to explain the observed relationship between $Q$ and $\eta_{\mathrm{H}_{2}}$

Pore initiation.-The physical background of pore propagation in silicon under conditions of (photo)anodic etching is rather well understood..$^{15,18,19}$ However, the fact that porous silicon can be formed photoanodically, starting from nearly atomically flat silicon surfaces with spatially homogeneous surface properties is not yet understood. ${ }^{24,27,28}$ The observation that porous etching is possible with p-type and n-type silicon crystals with large variations in doping density suggests that the primary initiation of pores has a chemical, rather than physical origin. We now investigate if pore initiation can be rationalized within the framework of the mechanism of photoanodic etching presented in previous sections. The essential steps of the mechanism can be summarized as follows

$$
\begin{aligned}
& \operatorname{Si}(\mathrm{O}) \frac{k_{0} p_{\mathrm{s}}}{+h_{\mathrm{VB}}^{+}} \operatorname{Si}(\mathrm{I}) \frac{k_{1}}{-e_{\mathrm{CB}}^{-}} \operatorname{Si}(\mathrm{II}) \frac{k_{2}}{-e_{\mathrm{CB}}^{-}} \operatorname{Si}(\mathrm{III}) \frac{k_{3}}{-e_{\mathrm{CB}}^{-}} \operatorname{Si}(\mathrm{IV})+\operatorname{Si}(\mathrm{O}) \\
& \downarrow k_{\mathrm{a}} \mathrm{s}_{1} \\
& \mathrm{Si}(\mathrm{IV})+\mathrm{H}_{2}+\mathrm{Si}(\mathrm{O})
\end{aligned}
$$

From a detailed kinetic study using intensity modulated photocurrent spectroscopy, Peter and co-workers found that the thermal injection of an electron from a surface bond of the $\mathrm{Si}$ (II) intermediate (reaction 3 ) is relatively slow, at least much slower than the injection of an electron from an $\mathrm{Si}(\mathrm{I})$ intermediate. ${ }^{14,17}$ Electron injection from the
Si(I) intermediate may involve a half-broken bond or an Si-SiHF radical species, while the $\mathrm{Si}$ (II) species already has two Si-F bonds. It is assumed here that the chemical oxidation of an $\mathrm{Si}(\mathrm{II})$ intermediate catalyzed by an $\mathrm{Si}(\mathrm{I})$ intermediate (the competitive reaction 5) is much faster than the electron injection (reaction 3 ) from the $\mathrm{Si}(\mathrm{II})$ intermediate into the conduction band. Since the catalyzed reaction 5 leads to a new $\mathrm{Si}(\mathrm{O})$ entity, a new $\mathrm{Si}(\mathrm{II})$ intermediate can be readily formed by reaction 1 (hole trapping) and reaction 2 (electron injection). This would imply that anodic dissolution proceeds much faster in a surface region round an $\mathrm{Si}(\mathrm{I})$ intermediate. Such an active region can be characterized by a radius which is determined by the diffusion length $L_{\mathrm{Si}(\mathrm{I})}$ of the mobile $\mathrm{Si}(\mathrm{I})$ intermediate. The diffusion length is defined as

$$
L_{\mathrm{S}(1)}=\sqrt{D_{\mathrm{Si}(1)} \times \tau_{\mathrm{Si}(1)}}
$$

where $D_{\mathrm{S}(\mathrm{I})}$ is the surface diffusion coefficient of the Si(I) intermediate and $\tau_{\mathrm{Si}(\mathrm{I})}$ is the lifetime, given by

$$
\tau_{\mathrm{Si}(\mathrm{I})}=\left(k_{\mathrm{a}} s_{2}+k_{1}\right)^{-1}
$$

Inhomogeneous etching will occur if the diffusion length of the $\mathrm{Si}(\mathrm{I})$ intermediate is smaller than the average distance between these intermediates

$$
\sqrt{D_{\mathrm{S}(1)} \times \tau_{\mathrm{Si}(1)}}<\frac{1}{\sqrt{s_{1}}}
$$

or, using Eq. 22, 12, and 13

$$
D_{\mathrm{Si}([)} \ll \frac{k_{1}}{j_{\mathrm{h}}^{\prime}}\left(k_{1}+\frac{k_{\mathrm{a}} k_{1} j_{\mathrm{h}}^{\prime}}{k_{1} k_{\mathrm{z}}+k_{\mathrm{a}} j_{\mathrm{h}}^{\prime}}\right)
$$

In the framework of our model, anodic dissolution occurs with formation of pores for anodic currents up to $j_{\mathrm{h}}=$ $2 e k_{1}^{2} / D_{\mathrm{Si}(\mathrm{I})}$. It would be interesting to investigate whether this maximum current density corresponds to the value which determines the region of porous silicon formation. ${ }^{18}$ This will, however, require a reliable value of the surface diffusion coefficient of the Si(I) intermediate. The model for pore initiation may be verified by measuring the initial density of pores as a function of the anodization current in the primary stages of porous etching. It should be possible, we believe, to perform these measurements using scanning tunneling microscopy of atomic force microscopy, or maybe other surface imaging techniques.

It can be concluded that the mechanism of porous etching, which we have proposed here for explaining the unique relationship between $\eta_{\mathrm{H}_{2}}$ and $Q$, may also provide an explanation for pore initiation. Since the basis for pore nucleation relies on (electro)chemical kinetics, the explanation pertains to both n-type and p-type silicon in $\mathrm{HF}$, independent of the doping density.

\section{Conclusions}

We have developed a kinetic scheme for the dissolution of silicon in HF containing solutions. From measurements on n-type silicon it has previously been found that both the photocurrent quantum efficiency $Q$ and the number of hydrogen molecules formed per absorbed photon, referred to as $\eta_{\mathrm{H}_{2}}$, are dependent on the light intensity. The kinetic analysis yields expressions for $Q$ and $\eta_{\mathrm{H}_{2}}$ which explain the experimental results. The key step in the kinetic scheme is a chemical reaction of an $\mathrm{Si}(\mathrm{II})$ surface intermediate with two protons to give an $\mathrm{Si}$ (IV) dissolution product and $\mathrm{H}_{2}$, a reaction which is catalyzed by a mobile surface intermediate $\mathrm{Si}(\mathrm{I})$. Based on the kinetic model a mechanism is proposed for the anodic dissolution of silicon in $\mathrm{HF}$ and the corresponding surface chemistry is discussed. The possible implications of this mechanism and, in particular, the autocatalytic step for the dissolution process and the resulting morphology are mentioned. We showed that this mechanism may provide an explanation based on chemi- 
cal kinetics, for initiation of pores during the anodic etching of silicon in $\mathrm{HF}$ solutions.

\section{Acknowledgment}

The work described here was supported by the Netherlands Foundation for Chemical Research (SON) with financial aid from the Netherlands Organization for Scientific Research (NWO).

Manuscript submitted Aug. 30, 1996; revised manuscript received Dec. 11, 1996.

\section{REFERENCES}

1. R. Memming and G. Schwandt, Surf. Sci., 4, 109 (1966).

2. M. Matsumura and S. R. Morrison, J. Electroanal. Chem., 147, 157 (1983).

3. H. Gerischer and M. Lübke, Ber. Bunsenges. Phys. Chem., 91, 394 (1987).

4. H. J. Lewerenz, J. Stumper, and L. M. Peter, Phys. Rev. Lett, 61, 1989 (1988)

5. H. Gerischer and M. Lübke, This Journal, 135, 2782 (1988).

6. L. M. Peter, D. J. Blackwood, and S. Pons, Phys. Rev. Lett., 62, 308 (1989).

7. J. Stumper, H. J. Lewerenz, and C. Pettenkofer, Phys. Rev. B, 41, 1592 (1990).

8. M. J. Eddowes, J. Electroanal. Chem., 280, 297 (1990).

9. L. M. Peter, A. M. Borazio, H. J. Lewerenz, and J. Stumper, ibid., 290, 229 (1990).

10. L. M. Peter, D. J. Blackwood, and S. Pons, ibid., 294, $111(1990)$

11. L. T. Canham, Appl. Phys. Lett., 57, 1046 (1990).

12. A. Venkateswara Rao, F. Ozanam, and J.-N. Chazalviel, This Journal, 138, 153 (1991).

13. V. Lehmann and U. Gösele, Appl. Phys. Lett., 58, 856 (1991).
14. J. Stumper and L. M. Peter, J. Electroanal. Chem., 309, 325 (1991).

15. X. G. Zhang, This Journal, 138, 3750 (1991)

16. C. Lévy-Clément, A. Lagoubi, R. Tenne, and M. Neumann-Spallart, Electrochim. Acta, 37, 877 (1992).

17. D. J. Blackwood, A. Borazio, R. Greef, L. M. Peter, and J. Stumper, ibid., 37, 889 (1992).

18. R. L. Smith and S. D. Collins, J. Appl. Phys., 71, R1 (1992).

19. P. C. Searson, J. J. Macauley, and F. M. Ross, ibid., 72, 253 (1992)

20. P. C. Searson, J. M. Macauley, and S. M. Prokes, This Journal, 139, 3373 (1992).

21. E. Propst and P. A. Kohl, ibid., 140, L78 (1993)

22. H. Gerischer, P. Allongue, and V. C. Kieling, Ber. Bunsenges. Phys. Chem., 97, 753 (1993).

23. C. Lévy-Clément, A. Lagoubi, and M. Tomkiewicz, This Journal, 141, 958 (1994).

24. St. Frohnhoff, M. Marso, M. G. Berger, M. Thönissen, $\mathrm{H}$. Lüth, and $\mathrm{H}$. Münder, ibid., 142, 615 (1995).

25. M. M. Rieger and P. A. Kohl, ibid., 142, 1490 (1995).

26. P. Allongue, C. H. de Villeneuve, L. Pinsard, and M. C. Bernard, Appl. Phys. Lett., 67, 941 (1995).

27. A. Valance, Phys. Rev. B, 52, 8323 (1995).

28. G. C. John and V. A. Singh, ibid, 52, 11125 (1995).

29. P. M. M. C. Bressers, J. W. J. Knapen, E. A. Meulenkamp, and J. J. Kelly, Appl. Phys. Lett., 61, 108 (1992)

30. L. T. Canham, W. Y. Leong, M. I. J. Beale, T. I. Cox, and L. Taylor, ibid., 61, 2563 (1992).

31. D. Vanmaekelbergh and W. P. Gomes, J. Phys. Chem., 94, 1571 (1990).

32. A. R. de Wit, D. Vanmaekelbergh, and J. J. Kelly, This Journal, 139, 2508 (1992).

33. B. H. Erné, D. Vanmaekelbergh, and I. E. Vermeir, Electrochim. Acta, 38, 2259 (1993).

34. G. H. Schoenmakers, R. Waagenaar, and J. J. Kelly, This Journal, 142, L60 (1995). 